\title{
Tri-trophic Plankton Models Revised: Importance of Space, Food Web Structure and Functional Response Parametrisation
}

\author{
H.I. Egilmez ${ }^{1}$, A.Yu. Morozov ${ }^{1,2 *}$ \\ ${ }^{1}$ Department of Mathematics, University of Leicester, Leicester, LE1 7RH, UK \\ ${ }^{2}$ Shirshov Institute of Oceanology, Moscow, 117997, Russia
}

\begin{abstract}
Revealing mechanisms of efficient top-down control in eutrophic ecosystems remains a long term challenge in theoretical ecology. In this paper, we revisit the role of environmental heterogeneity, food web structure and shape of the predator functional response in persistence and stabilization of a planktonic system with high nutrient supply. We consider a 1D vertically resolved tri-trophic planktonic food web composed of a primary producer, an intermediate predator and a highly mobile top predator, such as a system of phytoplankton, microzooplankton and copepods. We explore the realistic scenario in which the top predator is omnivorous, i.e. when copepods can feed both on phytoplankton and microzooplankton. We show that the interplay between heterogeneity of the environment due to for instance, a light gradient in the water column, and trophic interaction between species can result in an efficient top-down control which would otherwise be impossible in the corresponding well-mixed system. We also find that allowing the top predator to feed on the primary producer may dramatically impede the coexistence of the three trophic levels, with only two levels generally surviving. The coexistence of all three trophic levels within a wide range of parameters becomes possible only when the top predator exhibits active food source switching behaviour. We also show the phenomenon of bistability in the considered tri-trophic food web: a small initial amount of the top predator should lead to its extinction whereas introduction of a supercritical initial amount will eventually result in establishment of the population. The demonstrated mechanism of top-down control seems to be rather generic and might be a good candidate to explain stability in some other non-planktonic tri-trophic ecosystems.
\end{abstract}

Keywords and phrases: Integro-differential equations, top-down control, population persistence, stability, paradox of enrichment.

Mathematics Subject Classification: 47A75, 45K05, 92D40

\section{Introduction}

Plankton communities have always been an inspiring paradigm for modelling complex systems in ecology, and explaining the possibility of efficient top-down control of primary producers in planktonic ecosystems under eutrophication (i.e. ecosystems with high concentration in nutrients) ranks among the most

\footnotetext{
${ }^{*}$ Corresponding author. E-mail: am379@leicester.ac.uk
} 
intriguing problems. Simple models describing the interaction between zooplankton and phytoplankton in nutrient rich waters predict the emergence of large-amplitude population cycles eventually resulting in species collapse and extinction [50]. On the other hand, the empirical literature provides a large number of case studies where the density of phytoplankton remain relatively low even if the nutrient supply is high $[5,9,13,31]$. This apparent contradiction between theory and reality is known as the paradox of enrichment [50], and various mechanisms have been proposed to solve this long standing enigma $[2,25,40,42,51]$. The paradox of enrichment is not limited to planktonic ecosystems but is found in several other ecosystems including terrestrial agroecosystems [36].

Previously, non-homogeneous spatial distribution of organisms, as well as heterogeneity of the environment, in which species interactions take place, have been shown to promote top-down control in eutrophic ecosystems and potentially solve the paradox of enrichment $[37,39,44]$. For instance, in plankton ecosystems vertical gradients of resources such as nutrients or light may be an important factor in reducing and stabilizing large amplitude population cycles $[17,18]$. According to the reported scenario, stabilization requires some dispersal of predators (microzooplankton grazers), but the movement of organisms should be sufficiently slow, i.e. small-sized grazers should stay and feed close to the place where they were born, because fast diffusivity of grazers will destabilize the system. We can refer to this type of predator as a 'local' predator. Another recently found stabilizing mechanism involves the fast motion of predators (e.g. herbivorous mesozooplankton) across the entire euphotic zone of the water column and their preferential feeding in locations with high densities of prey $[21,37,39]$. On a demographic timescale the predator population should be described by an integral quantity such as the population size, since we cannot assign an organism to a particular spatial location. Therefore in this case the top-down control is considered to be caused by a 'global' predator.

In realistic planktonic food webs, both local and global types of grazers are usually present [46], which raises the question of how combining these two types can affect the stabilization and persistence of species. When taking into account fast and slow moving grazers, we can realistically assume that a typical plankton food web is tri-trophic $[27,28]$ : it includes producers such as phytoplankton, slow moving predators such as microzooplankton and fast displacing higher predators such as copepods. The stability of tri-trophic food chains including local and global predators has recently been explored by Lewis et al 2013 [35], where the authors found that primary producers can be controlled by grazers, although the phytoplankton density can 'bloom' and have their high values near the surface. However, these findings were obtained using some particular assumptions about the structure of the food web and the formulation of the functional response of the predator. In particular, it was assumed that the top predators can only consume intermediate grazers, and not the primary producers. Although copepods may indeed try to select nutritiously superior microzooplankton [30], there is a great body of evidence that phytoplankton can be also an important food source for them $[6,22,27,29,40]$. Therefore it would be more realistic to consider omnivorous copepods, even if their consumption of phytoplankton may be relatively small. In theoretical ecology such predation is known as 'intragild' predation, and it is a widespread phenomenon in food webs [45]. Another important assumption in [35] was that the spatial distribution of copepods followed info-chemicals released as a result of grazing of phytoplankton by microzooplankton, which may not always be correct [4].

In this paper, we reconsider these previous results concerning top-down control in tri-trophic eutrophic ecosystems in a heterogeneous environment under eutrophication, by investigating the realistic situation in which a fast moving top predator is omnivorous, and its spatial distribution follows the distribution of organisms of both lower trophic levels. We also explore the role of the parametrization of the functional response of the predators in the persistence and stability of the system. We demonstrate that the interplay between heterogeneity of the environment and the trophic interactions can result in a strong top-down control, which would be otherwise impossible in the same system without space. However, investigation of the model within realistic parameter ranges shows that consumption of phytoplankton by copepods generally results in a collapse of the ecosystem: microzooplankton and copepods cannot coexist together. The coexistence of the three trophic levels becomes possible only after introducing a particular assumption 
regarding the shape of the functional response of copepods - the functional response should exhibit active switching between food sources. We also find bistability in the system with omnivorous copepods: the successful establishment of copepods in the system requires there to be a supercritical initial number of them.

\section{Model equations}

We consider a tri-trophic planktonic food web which consists of phytoplankton $P$ and two types of predators: microzooplankton $M$ and larger predators, copepods $Z$. These take the role of the primary producer, intermediate predator and top predator in the system, respectively. The variable $h$ describes the depth in the water column; the trophic interactions take place in the upper euphotic zone between the surface $(h=0)$ and the base of this zone $(h=H)$. The model equations read as follows

$$
\begin{aligned}
\frac{\partial P(h, t)}{\partial t} & =D \frac{\partial^{2} P}{\partial h^{2}}+r \exp \left(-\phi h-\omega \int_{0}^{h} P(h) d h\right) P\left(1-\frac{P}{K}\right)-m P \\
& -f_{P_{1}}(P) M-f_{P_{2}}(P, M) \bar{Z} z(h), \\
\frac{\partial M(h, t)}{\partial t} & =D \frac{\partial^{2} M}{\partial h^{2}}+\gamma M f_{P_{1}}(P)-\mu M-f_{M}(P, M) \bar{Z} z(h), \\
\frac{d \bar{Z}(t)}{d t} & =\frac{\bar{Z}}{H} \int_{0}^{H}\left(\epsilon_{P} f_{P_{2}}(P, M)+\epsilon_{M} f_{M}(P, M)\right) z(h) d h-\delta \bar{Z},
\end{aligned}
$$

where $P(h, t)$ and $M(h, t)$ are the species densities at depth $h$ and time $t$, whereas $\bar{Z}(t)$ is the space average density of copepods over the entire euphotic zone; $z(h)$ is the relative local density of copepods, so the local density of copepods at depth $h$ is given by the product of $\bar{Z}$ and $z(h)$.

The model parameters have the following meaning: $r$ is the maximum growth rate and $K$ is the carrying capacity of the phytoplankton, which we include instead of explicitly modelling depletion of nutrients in the water. The growth rate of the phytoplankton is proportional to the light intensity (we assume that the light irradiance is not very high and there is no growth inhibition, see [20]), which exponentially decreases with depth due to (i) absorbtion by water (which is described by the absorption coefficient $\phi$ ) and (ii) self-shading by the phytoplankton located above the given depth (which is described by an integral term, with the self-shading coefficient $\omega$ ). The parameters $m, \mu$ and $\delta$ are the mortality rates of phytoplankton, microzooplankton and copepods, respectively. The function $f_{P_{1}}$ is the functional response of microzooplankton; $f_{P_{2}}$ and $f_{M}$ describe food consumption by copepods. The conversion of grazed phytoplankton into new biomass of microzooplankton is described by $\gamma$, whereas phytoplankton and microzooplankton conversion efficiency of copepods are described by $\epsilon_{P}$ and $\epsilon_{M}$, respectively.

The plankton system is subject to zero-flux boundary constraints to prevent plankton leaving the considered part of the water column:

$$
\begin{aligned}
& \frac{\partial P(0, t)}{\partial h}=\frac{\partial P(H, 0)}{\partial h}=0 \\
& \frac{\partial M(0, t)}{\partial h}=\frac{\partial M(H, 0)}{\partial h}=0 .
\end{aligned}
$$

To proceed further, we need to parameterise the functional responses of the zooplankton. The functional response of microzooplankton should depend only on the density of phytoplankton. Using the conventional approach, we shall assume the following parametrisation [26]

$$
f_{P_{1}}=\frac{g P}{k_{1}+P}
$$

where $k_{1}$ is the half saturation constant and $g$ is the maximal intake rate. 
For comparison with the model with an omnivorous top predator, we shall first consider the simplest scenario, in which copepods only graze on microzooplankton. In this case, the functional responses become

$$
f_{P_{2}}=0, f_{M}=\beta \frac{M}{k_{2}+M},
$$

where $k_{2}$ is the half saturation constant and $\beta$ is the maximal intake rate.

Parametrization of the functional response of copepods depending on two food sources is a more complicated issue. Overall, there exist a large number of possible parameterisations, each of which has its biological rationale (see [26], [41] and [48]). In this paper, we consider two different types of multi-prey functional responses: (i) no switching response and (ii) response with switching.

A no-switching functional response is defined as one where the relative preference of the resources is constant [26]. Here we use the following well-known parametrisation of a no-switching functional responses known as the Disk (Holling type II) equation [22,43]:

$$
f_{P_{2}}=\beta_{1} \frac{P}{k_{2}+P+M}, f_{M}=\beta_{2} \frac{M}{k_{2}+P+M},
$$

where $\beta_{1}$ and $\beta_{2}$ are the attack rates, $k_{2}$ is the half saturation coefficient.

In the functional response with active switching, the food preference of the predator depends on the densities of the two food resources. In this paper, we use the recently suggested parametrisation known as the 'kill-the-winner' functional response given by [56]

$$
f_{P_{2}}=\beta_{1} \frac{P^{2}}{P^{2}+M^{2}} \frac{P}{k_{2}+P+M}, f_{M}=\beta_{2} \frac{M^{2}}{P^{2}+M^{2}} \frac{M}{k_{2}+P+M},
$$

where $\beta_{1}, \beta_{2}$ and $k_{2}$ have the same meaning as in (2.4). The formal derivation of parametrization (2.5) is discussed in detail in [56]. Note that the main difference between responses (2.4) and (2.5) is that active switching causes a substantial drop in resource consumption when the relative abundance of this resource is low.

We define Models 1,2 and 3 according to the functional responses of the top predator used in each of them: in Model 1 the copepods are not omnivorous, i.e. the functional response is given by (2.3). In Model 2, the functional response is a no-switching functional response parameterized by (2.4). Model 3 is characterized by a functional response with resource switching which is expressed by $(2.5)$.

Both the primary producers and microzooplankton have limited swimming abilities and are subject to a vertical turbulent diffusion, $D$. This is described by the corresponding diffusion terms. On the other hand, copepods are highly mobile organisms with swimming speeds which allow them to resist turbulence and quickly adjust their vertical position. Since active movement of copepods occurs on a fast scale compared to their demographic scale, we cannot assign an individual copepod to a fixed horizontal layer in the water column. Following [39], we describe the spatial distribution of copepods based on the relative distribution $z(h)$, which is the local density of copepods divided by their average density $\bar{Z}$. Based on previous empirical demonstrations [21,34,38,39], we can assume that the relative distribution of copepods follows the distribution of resources across the space, i.e.

$$
z(h)=\frac{\eta_{1} P(h)+\eta_{2} M(h)}{\eta_{1} \bar{P}+\eta_{2} \bar{M}},
$$

where $\bar{P}$ and $\bar{M}$ are the space average densities of $P$ and $M$, respectively. Note that for simplicity, the weights in (2.6) are chosen to be the same as in the above functional responses to reduce the number of independent parameters. This assumption is not critical and one can replace $\eta_{i}$ with some independent parameters. Note that the above expression for $z(h)$ only holds for Models 2,3. For Model 1, one should remove in this expression the density $P$ since according to the assumptions of this model, copepods do not feed on the primary producers. Alternatively, one can formally set $\eta_{1}=0$ for Model 1 . 


\begin{tabular}{|l|l|l|l|l|}
\hline Parameters & Definition & Units & Fixed values & Ranges \\
\hline $\mathrm{r}$ & Phytoplankton intrinsic growth rate & $d^{-1}$ & 1.5 & $0.1-2[16]$ \\
\hline $\mathrm{K}$ & Phytoplankton carrying capacity & $\mu g C l^{-1}$ & 2000 & $50-$ inf $[39]$ \\
\hline$\phi$ & Light attenuation coefficient & $m^{-1}$ & 0.05 & $0.005-0.15[3]$ \\
\hline$\omega$ & Self-shading coefficient & $1 /\left(m \mu g C l^{-1}\right)$ & 0.002 & $0.0005-0.005[3]$ \\
\hline$g$ & Microzooplankton grazing rate & $d^{-1}$ & 7.5 & $2-12[17]$ \\
\hline$\beta_{1}$ & Copepods grazing rate & $d^{-1}$ & 1.4 & $0.6-1.4[16,29]$ \\
\hline$\beta_{2}$ & Copepods predation rate & $d^{-1}$ & 1.4 & $0.6-1.4[29,39]$ \\
\hline$k_{1}$ & Half saturation constant & $\mu g C l^{-1}$ & 20 & $20-100[29,39]$ \\
\hline$k_{2}$ & Half saturation constant & $\mu g C l^{-1}$ & 60 & $20-100[29,39]$ \\
\hline$\gamma$ & Microzooplankton grazing efficiency & - & 0.3 & $0.15-0.64[17]$ \\
\hline$\epsilon_{P}$ & $\begin{array}{l}\text { conversion efficiency- phytoplankton } \\
\text { to copepods }\end{array}$ & - & 0.35 & $0.2-0.8[17,28]$ \\
\hline$\epsilon_{M}$ & $\begin{array}{l}\text { conversion efficiency- microzooplankton } \\
\text { to copepods }\end{array}$ & - & - & \\
\hline$m$ & Phytoplankton mortality & $d^{-1}$ & 0.7 & $0.2-0.8[17,28]$ \\
\hline$\mu$ & Microzooplankton mortality & $d^{-1}$ & 0.02 & $0-0.3[24]$ \\
\hline$\delta$ & Copepods mortality & $d^{-1}$ & 0.05 & $0.01-0.1[8]$ \\
\hline$D$ & Diffusion coefficient & $m^{2} d^{-1}$ & 1 & $0.01-0.15[8,18]$ \\
\hline
\end{tabular}

TABle 1. Parameters definitions, units, fixed values and possible ranges. The corresponding references are provided

Note that there is some indication in the literature that the conversion efficiency of copepods feeding on microzooplankton is often higher than when feeding on phytoplankton $[28,54]$. However, the precise ratio between $\epsilon_{P}$ and $\epsilon_{M}$ is generally unknown. In this paper, we assume that $\epsilon_{P}=0.5 \epsilon_{M}$ in most of simulations. However, we also consider the scenario when $\epsilon_{P}=\epsilon_{M}$. Note that variation of the ratio $\epsilon_{P} / \epsilon_{M}$ does not greatly affect the main qualitative results of the paper.

The parameter values and ranges considered here are take from the literature; they are summarized in Table 1. We consider the size of the euphotic zone to be $H=80 \mathrm{~m}$. The initial conditions for numerical simulations assumed uniform distributions of phytoplankton and microzooplankton. The unit of plankton density is chosen as $\mu \mathrm{gCl}^{-1}$. The models were analyzed numerically using an explicit finite-difference method, where the integral was evaluated using the trapezoidal rule [32].

\section{Results}

Firstly, we briefly consider Models 1,2 and 3 in the case of a well-mixed system, i.e. assuming the ecosystem to be homogeneous. This will allow us to better understand the role of spatial heterogeneity in persistence and stability. The model equations of the corresponding well-mixed systems are formally obtained by removing the diffusion terms and setting $\phi=0, \omega=0$ and $z(h)=1$ (see Appendix A). The resultant model then becomes a system of ODEs and can be studied analytically. We found nontrivial stationary states for all considered models (for Model 3 analytical methods were combined with numerical techniques) and then analyzed the stability of those stationary states within the considered parameter ranges. Since the phytoplankton growth rate in the spatial model varies across the depth, in the corresponding non-spatial model we covered a large range of values of $r$ to make our comparison fair $(0<r<2)$. For none of the non-spatial models were we able to find a single set of parameters where a non-trivial stationary state was stable (within the considered parameter range, see Appendix A). Thus stable co-existence of all three trophic levels within a realistic parameter range is impossible in the case of a well-mixed ecosystem. Our numerical simulations also revealed large amplitude oscillations, where the species densities drop to very low values, which would result in eventual population extinction (result not shown here). 
Including spatial heterogeneity, however, can result in system stabilization and allow coexistence of all three trophic levels. We shall consider Models 1,2 and 3 separately.

In Model 1, the coexistence of three trophic levels is possible for a supercritical conversion efficiency of copepods $\epsilon_{M}$. Variation of the average species densities are shown in the bifurcation diagrams in Fig.1. This and all other diagrams have been constructed numerically, by plotting averaged over the euphotic zone population densities for $t>2000$, after the initial transients died out. At very low $\epsilon_{M}$ $\left(\epsilon_{M}<0.058\right)$ copepods cannot get establish in the system and the phytoplankton density is kept low by microzooplankton grazing. For some higher values of $\epsilon_{M}\left(0.058<\epsilon_{M}<0.25\right)$, all three trophic levels can coexist, although species densities exhibit temporal oscillations. For $\epsilon_{M}>0.25$ stable coexistence of all species become possible. An example of the distribution of phytoplankton and microzooplankton across the water column is shown in Fig.2 constructed for $\epsilon_{M}=0.4$. One can see from the figure that although the system is stabilized, the local density of primary producers may achieve very high values near the surface, which signifies an intensive plankton bloom. The model also exhibits bi-stability, which is explained at the end of the section.
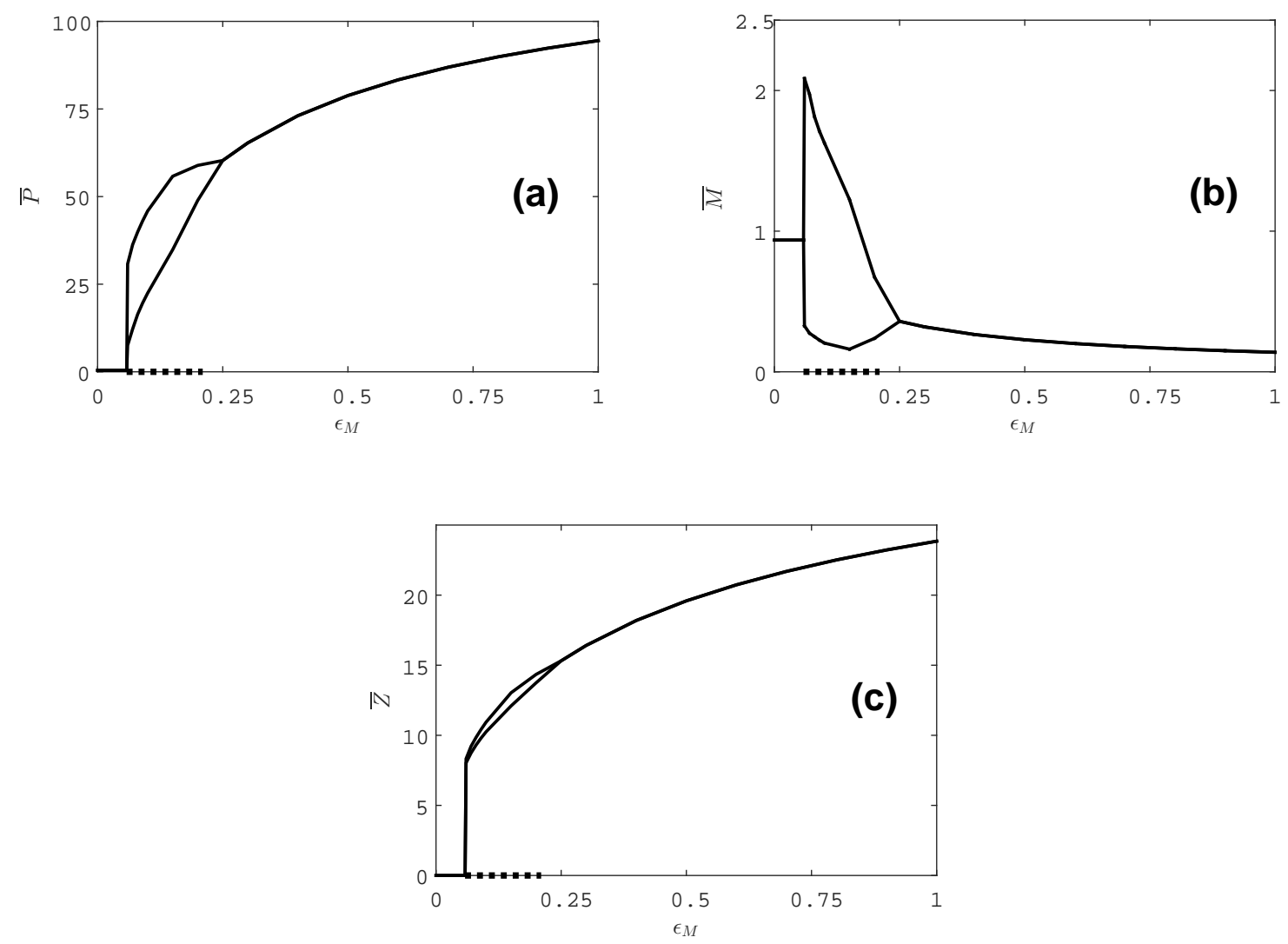

FiguRE 1. Spatially average densities of phytoplankton (a), microzooplankton (b) and copepods (c) in Model 1 plotted against microzooplankton conversion efficiency to copepods $\epsilon_{M}$. The other parameters are given in table 1 (fixed values). In the case of periodic oscillations, the maximal and the minimal densities are shown in the figure. The dashed segment on the horizontal axis denotes the range of bistability, where copepods establishment depends on initial density. 

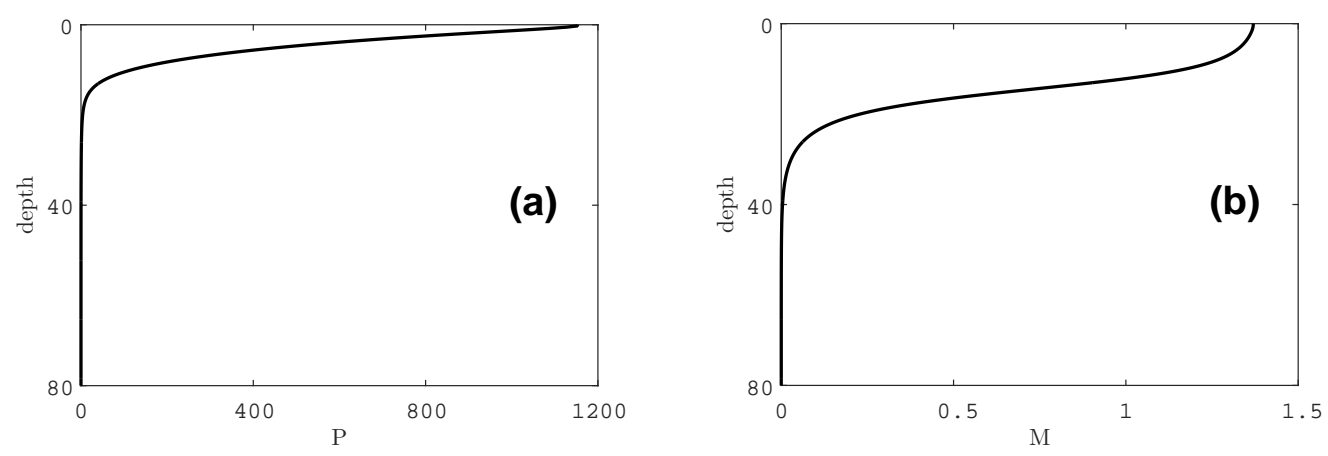

Figure 2. Vertical distributions of phytoplankton (a) and microzooplankton (b) in Model 1 shown for $\epsilon_{M}=0.7$. The other parameters are the same as in Fig.1

In Model 2, where the top predator is assumed to be omnivorous, we found that the coexistence of the three trophic levels to be very restricted. This can be seen from the bifurcation diagram in Fig.3. We always consider that $\epsilon_{P}=0.5 \epsilon_{M}$. At low $\epsilon_{M}$, copepods cannot get established in the system and the primary production is controlled by microzooplankton. The corresponding vertical distribution of the species are shown in Fig.4a. For $\epsilon_{M}>0.12$ the predation of microzooplankton by copepods results in extinction of the intermediate trophic level with only the primary producer and the top predator surviving in the long term. The vertical profile of phytoplankton is presented in Fig.4b (the distribution of copepods follows that of $p(h)$ ). The coexistence of three trophic levels is possible only within a small rage of parameters centred at $\epsilon_{M}=0.11$ : a small increase/decrease of this parameter will result in extinction of either the second or third trophic level. The spatial distributions of all species are shown in Figs.4c,d. A particularly interesting feature here is the unimodal vertical profile of microzooplankton, with a maximum close to $h=18 \mathrm{~m}$, whereas the density of phytoplankton exhibits a monotonous decrease with depth. This observation can be explained by the fact that near the surface the density of copepods is so high (due to high concentrations of phytoplankton) that they overgraze the microzooplankton to local extinction. The grazing pressure by copepods drops with depth due to decrease in their own density because the concentration of phytoplankton drops with depth, and this allows microzooplankton to survive at some intermediate depths. The model also exhibits bi-stability, which is explained at the end of the section.

To verify that an omnivorous top predator largely hinders the coexistence of all species in the given tri-trophic food web, we have constructed a larger number of bifurcation diagrams. An insightful example is the set of diagrams in the $\left(\beta_{1}, \beta_{2}\right)$ plane shown in Fig.5. The diagrams are constructed for $\epsilon_{M}=0.1$, $\epsilon_{M}=0.12, \epsilon_{M}=0.14, \epsilon_{M}=0.2$ and $\epsilon_{P}=0.5 \epsilon_{M}$. One can see that the coexistence of all three trophic levels (denoted by green circles) is only possible within a rather narrow domain in the $\left(\beta_{1}, \beta_{2}\right)$ plane: a small fluctuation in parameters would eventually result in extinction of a trophic level, either the intermediate or top predator.

In Model 3, where the top predator is omnivorous but exhibits active switching, the coexistence of all trophic levels is restored. A typical bifurcation diagram is shown in Fig.6. In the case where $\epsilon_{M}$ $\left(\epsilon_{P}=0.5 \epsilon_{M}\right)$ is large enough to allow the copepods to survive $\left(\epsilon_{M}>0.17\right)$, the coexistence of all species is possible in a stable mode: no oscillations are observed. Interestingly, the corresponding vertical distribution of primary producers (Fig.7) demonstrates much lower phytoplankton densities near the surface as compared to Model 1 (see Fig.2), with the difference being more than 3 orders of magnitude, i.e. no bloom is observed. Our extensive numerical simulations show that stable coexistence of all species is observed within a wide range of parameters. In particular, variation of $\left(\beta_{1}, \beta_{2}\right)$ within the same ranges as in Fig.5 will not result in extinction of any of the trophic level, so the corresponding diagrams would 

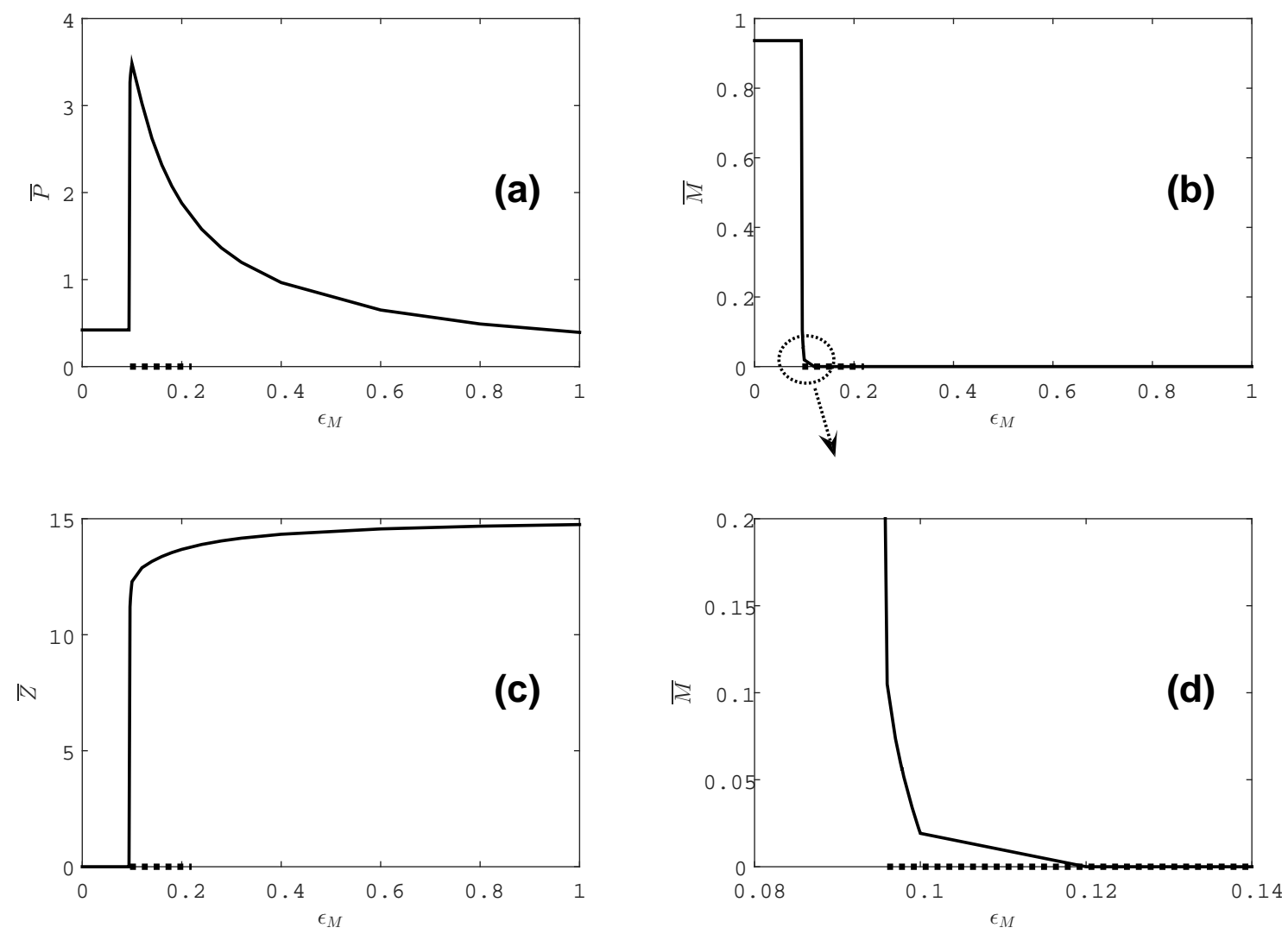

FiguRE 3. Spatially average densities of phytoplankton (a), microzooplankton (b) and copepods (c) in Model 2 are plotted against conversion efficiency of microzooplankton to copepods $\epsilon_{M}$. Diagram (d) is a zoom of diagram (b). $\epsilon_{P}=0.5 \epsilon_{M}$ and the other parameters are given in table 1 (fixed values). The dashed segment on the horizontal axis denotes the range of bistability, where copepods establishment depends on initial density.

be entirely filled with circles. Thus, active switching of the top predator maintains the entire tri-trophic food web structure.

We have explored the effect of the key parameters in Model 3 on the equilibrium species densities. Fig. 8 shows the dependence of the average densities $\bar{P}, \bar{M}$ and $\bar{Z}$ on the parameters $\delta, \phi$ and $\omega$, which are the mortality rate of copepods, the water absorption of coefficient and the self-shading coefficient, respectively. A gradual increase of $\delta$ (due to predation by fish, for instance) would weaken the top predator, resulting in a decrease in its density. This will translate itself into an increase of the densities of the two lower trophic levels. Beyond a critical value of $\delta$ the top predator cannot survive and the system becomes a two-level system consisting of the primary producer and the intermediate predator, microzooplankton. A gradual increase of $\phi$ (e.g. due to turbidity of water) or self shading coefficient $\omega$ would cause a decrease of the average densities of all species. This behaviour can be explained by the fact that an increase in water absorption or the densities of total species results in a drop of the density of the primary producer, which in turn results in a drop of the density of top predator. Due to a decrease of the trophic pressure exserted by the copepods and microzooplankton. 

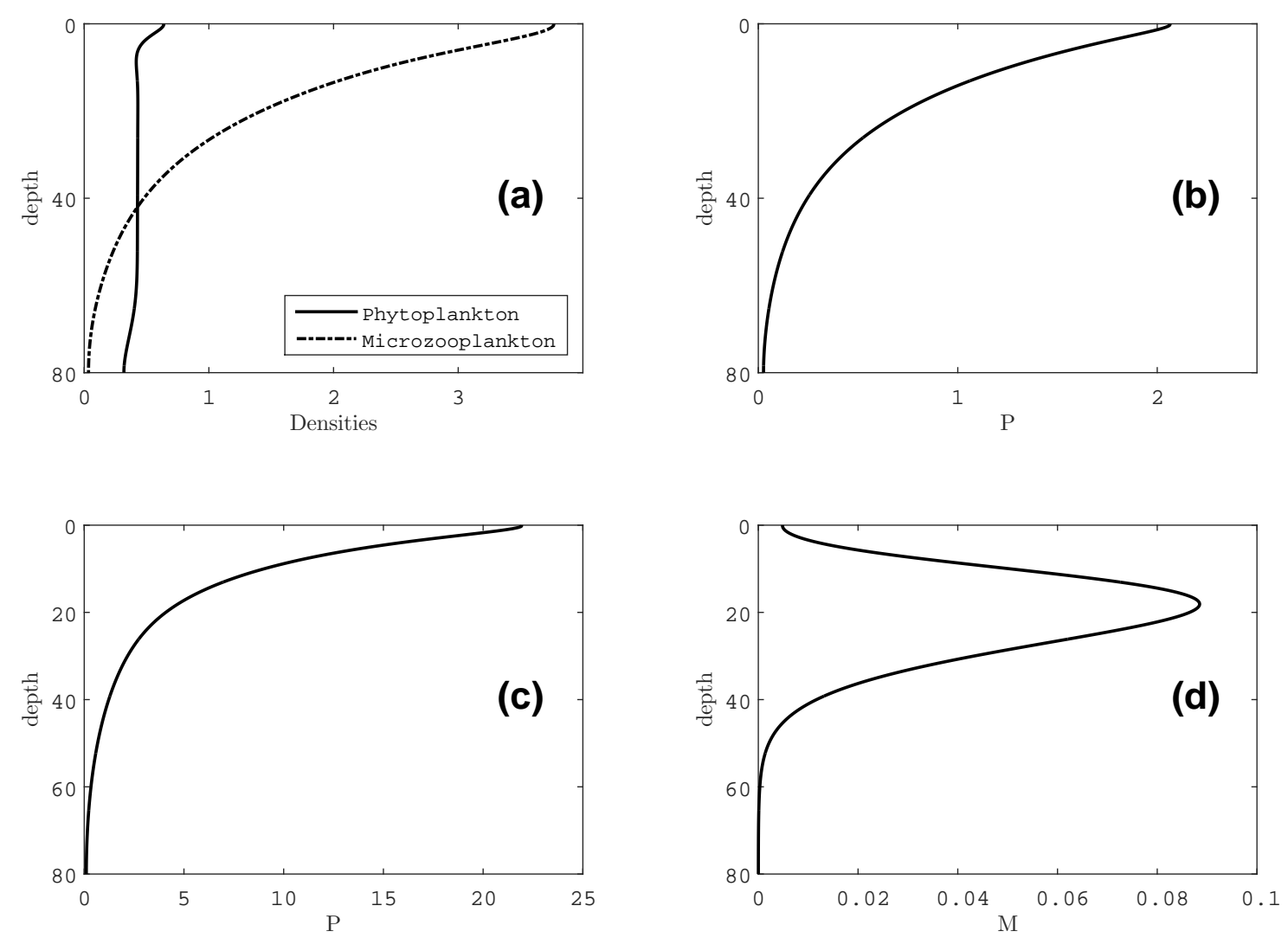

FiguRe 4. Vertical distribution of phytoplankton and microzooplankton in Model 2 according to different scenarios. (a) Vertical distribution of phytoplankton and microzooplankton in the absence of copepods $\left(\epsilon_{P}=0.05, \epsilon_{M}=0.1\right.$ and the initial copepods density $\bar{Z}_{0}=1$ ); (b) vertical distribution of phytoplankton in the absence of microzooplankton $\left(\epsilon_{P}=0.4\right.$ and $\left.\epsilon_{M}=0.7\right)$; (c), (d) vertical distribution of phytoplankton and microzooplankton in the case where the coexistence of the three trophic levels is possible , respectively. $\epsilon_{P}=0.05, \epsilon_{M}=0.1$, the initial copepods density $\bar{Z}_{0}=15$ and the other parameters are given in table 1 (fixed values).

Interestingly, in all of the three considered tri-trophic models, we found an interesting phenomenon of bistability. In Figs 1,3 and 6, the bistability range is denoted by a thick dashed line. For some intermediate values of $\epsilon_{P}$ and $\epsilon_{M}$, a successful establishment of the top predator depends on its initial amount in the system. At low initial density the copepods will go extinct (in this case the densities of $P$ and $M$ are the same as for $\left.\epsilon_{P}=\epsilon_{M}=0\right)$, whereas a supercritical initial $\bar{Z}$ can allow the population to persist. The range of bistability was obtained numerically by varying initial value of $\bar{Z}$ within a large interval.

The existence of bistability can be explained as follows. In the case where the initial amount of top predator is low and the system becomes essentially two-component, including only phytoplankton and microzooplankton. As a result, the vertical distributions of $P$ and $M$ become such that entire food consumption by rare copepods in the water column is smaller than the mortality rate, i.e. their per capita growth rate become negative. As a result, the population of copepods should unavoidably go extinct. On the other hand, a large initial amount of copepods transform the vertical distribution of $P$ 

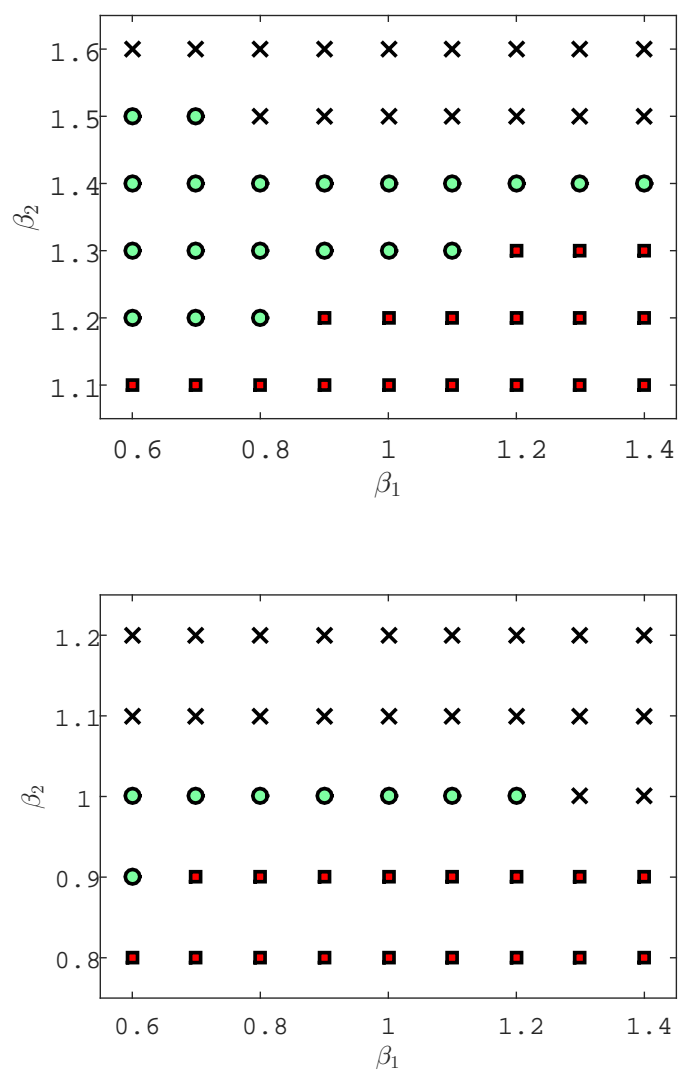

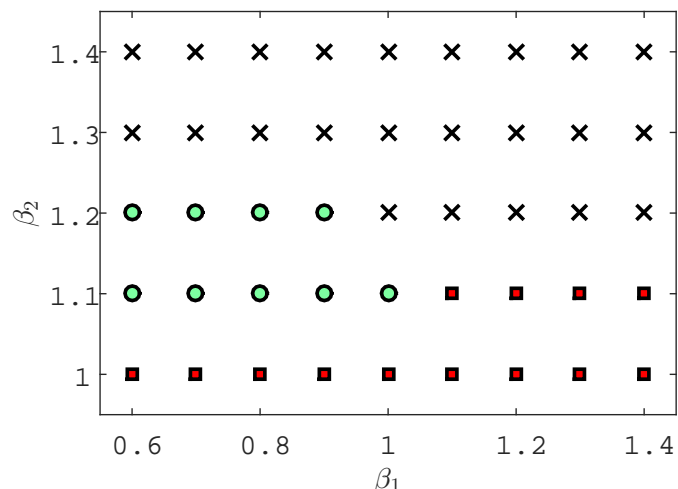

(b)

(c)

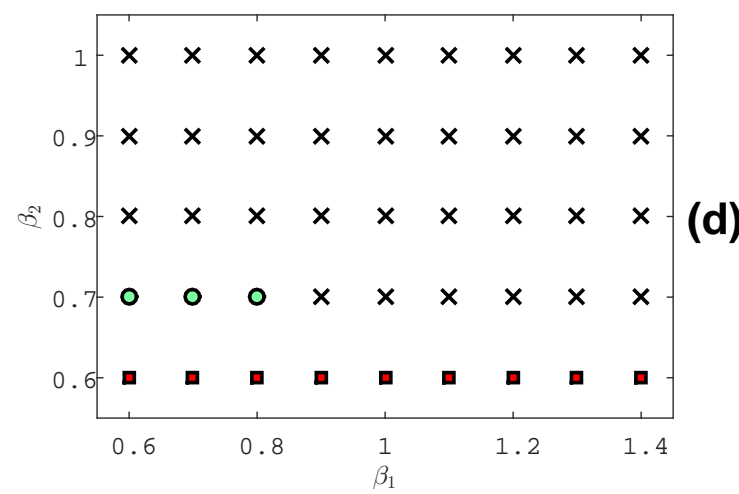

FiguRE 5. Bifurcation diagrams in the $\beta_{1}-\beta_{2}$ plane showing the outcome of interactions between the trophic levels for values of $\epsilon_{M}=0.1,0.12,0.14$, and 0.2 respectively. $\epsilon_{P}=0.5 \epsilon_{M}$ and the other parameters are given in table 1 (fixed values). Circles denote coexistence of all trophic levels. Crosses correspond to the coexistence of phytoplankton and copepods, with microozooplankton being extinct. Red squares denote the coexistence of phytoplankton and microzooplankton, with copepods being extinct.

and $M$ in such way that overall food intake becomes larger as compared to the system where copepods are rare. For instance, in Models 2 and 3, where copepods are allowed to feed on phytoplankton, the increase in their overall food consumption is mainly because of the release of phytoplankton from the overgrazing by microzooplankton. On the other hand, in Model 1 , for a large initial amount of $\bar{Z}$, the increase in the overall food intake of copepods is due to re-shaping the distribution of microzooplankton, by increasing the spatial gradients of $M(h)$. As a result, feeding of copepods takes place mainly at depths with the highest densities of microzooplankton, avoiding locations with low food density. We do not show here the corresponding graphs for the sake of brevity.

\section{Discussion and conclusions}

Despite the large body of literature on stability in eutrophic ecosystems that has been published since the seminal paper by Rosenzweig [49], finding reliable mechanisms of efficient top-down control is still in the focus of theoretical ecology. The main challenge in the resolution of this paradox is that there may exist a large number of stabilization mechanisms which vary from ecosystem to ecosystem [51]. On the 

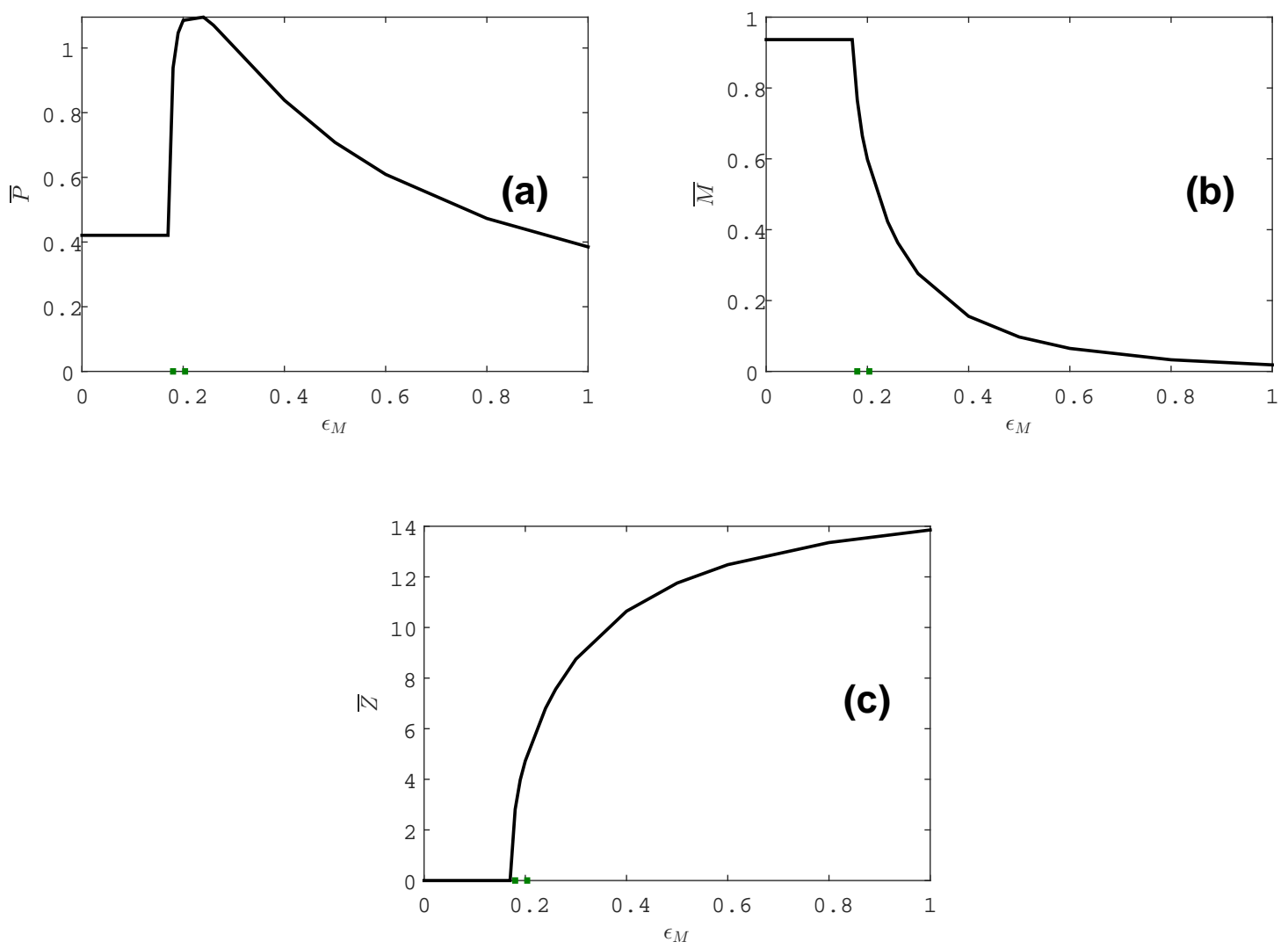

FiguRE 6. Spatially average densities of phytoplankton (a), microzooplankton (b) and copepods (c) in Model 3 are plotted against the copepod conversion efficiency $\epsilon_{M} ; \epsilon_{P}=$ $0.5 \epsilon_{M}$ and the other parameters are given in table 1 (fixed values). The dashed segment on the horizontal axis denotes the range of bistability, where copepods establishment depends on initial density.

other hand, several different mechanisms can be present in the same ecosystem. For instance, structuring in the primary producer population and spatial heterogeneity, which have been reported to be stabilizing factors (see $[21,39,40]$ ), can simultaneously affect top-down control. The general consensus in tackling the paradox of enrichment is that the initial predator-prey model suggested by Rosenzweig [49] is too simplistic and we need to include more realistic features to adequately represent our ecosystems.

Plankton communities in the ocean or deep lakes possess a combination of particular features which should be taken into account when explaining top-down control in such systems. Firstly, there is always a pronounced resource gradient, i.e. light, for the primary producers, phytoplankton [46]. Secondly, the light gradient in the water column is not static but is highly dynamic due to self-shading by the primary producers, which usually acts as a strong stabilizing factor [40]. Thirdly, some plankton grazers such as copepods can be highly mobile in the vertical direction and quickly adjust their location to feed at depths with high food density [38], whereas other grazers, such as microzooplankton, have much more limited dispersal abilities and usually feed near the locations where they are born. Finally, interaction between grazers is complex since higher trophic level can be omnivorous and consume both intermediate grazers and primary producers [53], which is known as an intraguild or intra-trophic predation [45]. Note that 


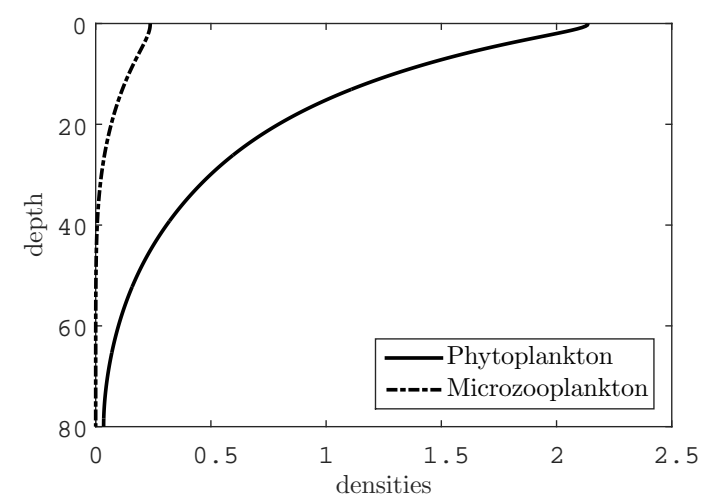

Figure 7. Vertical distributions of phytoplankton and microzooplankton in Model 3 shown for $\epsilon_{M}=0.7 . \epsilon_{P}=0.5 \epsilon_{M}$ and the other parameters are given in table 1 (fixed values).
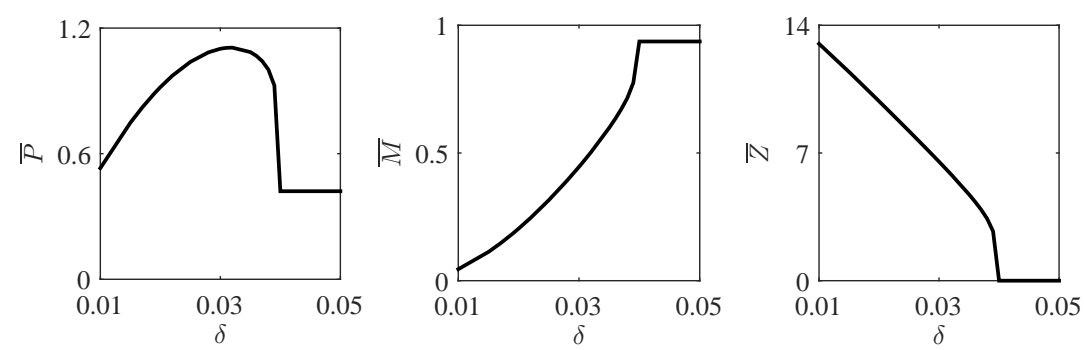

(a)
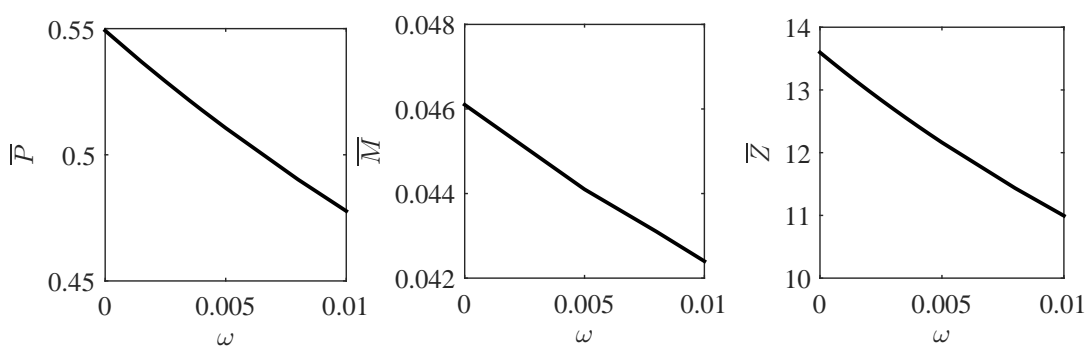

(b)
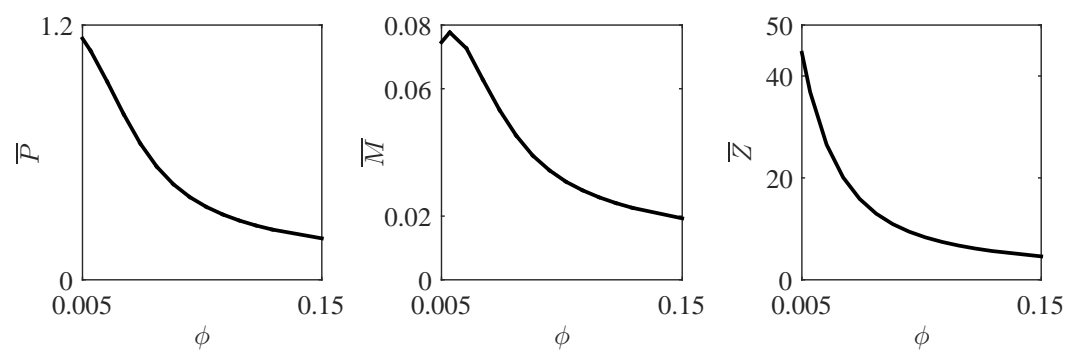

(c)

FiguRE 8. Bifurcation diagrams showing the average species densities in Model 3 as functions of key parameters. In each diagram, the other parameters are fixed as indicated in table 1 (fixed values). 
the existence of different time scales in planktonic ecosystems may require the use of models based on integro-differential equations [21].

In the current paper, we revisit the dynamics of a generic tri-trophic plankton model, taking into account the previously noted particularities. In our study, we assume the environment to be eutrophic, thus neglecting depletion of nutrients in the water. The constructed tri-trophic plankton model is still parsimonious but includes realistic assumptions on its spatial and trophic structure. To cover different possible scenarios we considered both an omnivorous and non-omnivorous top predator as well as different types of functional responses (both with and without active switching between food sources).

Our study shows that including space in tri-trophic interactions results in stabilization of the system, which would be impossible in the well-mixed case. This confirms some previous findings on the crucial role of space in the top-down control and stabilization of eutrophic ecosystems $[18,21,39,44]$. On the other hand, our results (covered by Model 2) show that making the realistic assumption of an omnivorous top predator (copepods) can be result in collapse of one of the trophic levels (either the microzooplankton or mesozooplankton). This occurs in the case where the functional response of copepods is a non-switching response, such as the classical Disk (Holling II) equation [26], as the parametric range of coexistence between the species in this case is so narrow that it should be considered as unrealistic (see Figs. 3,5). Introducing the strong assumption of active switching behaviour of copepods (covered by Model 3) can result in the coexistence of the entire food web with the density of primary producers remaining low (see Fig.7). This conclusion is comparable with the main results of [28], who found that active switching promotes coexistence of microzooplankton and copepods. Interestingly, some experimental works have also confirmed that active switching of copepods can stabilize a tri-trophic planktonic system [27], although these experiments were conducted on such a short time scale that the population dynamics of copepods were neglected.

Empirical observations in the ocean and lakes reveal coexistence between phytoplankton, microzooplankton and mesozooplankton without apparent extinction of any trophic level $[17,27,46]$. Based on our investigations of Models 1,2,3, this signifies that one should expect to see active switching behaviour in the feeding of copepods. Indeed, the use of a non-switching (Holing type II) functional response results in a collapse of either copepods or microzooplankton in models which, is not observed in nature. The existing experimental data provide evidence that some copepods (e.g. Acartia spp.) display a functional response with active switching between microzooplankton and phytoplankton [15,28,33]. This can be explained by the existence of two different feeding strategies of grazers: ambush feeding on mobile prey and suspension feeding by filtering of slow moving prey $[7,10,55]$. On the other hand, some other highly abundant copepod genera (e.g. Calanus, Para- and Pseudocalanus) do not exhibit active switching because they implement filter feeding most of the time $[27,55,57]$. Since in a realistic situation we have a mixture of different genera in the mesozooplankton compartment, the question of which type of functional response we should implement in a generic plankton model is still a matter of debate.

The question of why a particular copepod genera follows a particular type of functional response is fundamental to zooplankton ecology, with several different approaches proposed. For instance, based on the generic principles of evolution theory, one can suggest that the current functional responses are the result of long term natural selection. For instance, a functional response with active switching could be more beneficial for the entire population of copepods than a simple Monod-like (Holling type II) response, and the individuals using the more efficient response should over-compete the other copepod individuals. In this paper, we try to gain some preliminary insight into this complicated problem by modelling the outcome of the competition between two copepods species having different types of functional response. Namely, we considered the same tri-trophic model (4), where the top predator population $\bar{Z}$ consists of two subpopulations $\bar{Z}_{1}$ and $\bar{Z}_{2}$, each having a different functional response. We considered the three following combinations: (i) $\bar{Z}_{1}$ is non-omnivorous and $\bar{Z}_{2}$ is omnivorous with a non-switching functional response; (ii) $\bar{Z}_{1}$ is non-omnivorous and $\bar{Z}_{2}$ is omnivorous having a functional response with switching; (iii) $\bar{Z}_{1}$ is omnivorous with a non-switching functional response and $\bar{Z}_{2}$ is omnivorous having a functional 
response with switching. In each case, we assumed the same half saturation constants and the maximum consumption rates for the functional responses of both subpopulations of copepods.

Numerical simulations showed the following competition outcomes. Populations of omnivorous copepods (with or without active switching) always over-compete the population with a single source functional response. Thus, to have a supplementary food source seems to be beneficial for copepods. On the other hand, competition between omnivorous copepods with and without active switching results in the coexistence of both populations. This occurs mainly because intensive grazing by the subpopulation with the non switching functional response eradicates the intermediate trophic level (microzooplankton) and the resultant system becomes a two-level one. In the case where the maximal consumption rates are different, the winner will be the subpopulation of the copepods having the largest $\beta_{1}$ and $\beta_{2}$. Thus, our preliminary results indicate that a functional response either with or without switching may be selected. Note that in our simple competition analysis we neglected any potential cost of active switching, and morphological aspects which may not allow certain species to use different strategies. A more thorough analysis should be done elsewhere.

When investigating the models, we found the existence of alternative states in the system depending on the initial species densities: for some intermediate values of copepod efficiencies $\epsilon_{P, M}$ the models exhibit bi-stability. Namely, the establishment of copepods in the ecosystem is only possible for a sufficiently large initial population size, whereas a small initial amount of copepods will go extinct. This phenomenon is similar to the well-known strong Allee effect in ecology, when a drop of the population size below a certain critical threshold signifies its imminent extinction $[12,14]$. The Allee effect can be the result of a large variety of different ecological mechanisms [52]. In our case, initial supercritical amount of copepods is required to prevent of phytoplankton from overgrazing by microzooplankton or altering the vertical distribution of microzooplankton to enhance the overall intake rate by copepods (such as in Model 1). Interestingly, bistability in vertically distributed plankton ecosystems has been found in some other models [47].

To conclude, we have found that stability in a spatial tri-trophic plankton system is possible even for an unlimited nutrient load. This solves the paradox of enrichment for planktonic systems having a pronounced vertical depth, although for a sufficiently small $H$ the system becomes well-mixed and topdown control becomes impossible. The coexistence of the three trophic levels requires active switching behaviour by the copepods. We should emphasize, however, that efficient control of phytoplankton can occur in the case where only one type of grazer is present: a 'local' slow moving predator, such as microzooplantkon, or a fast moving 'global' predator, such as copepods. In other words, the simultaneous presence of two predators does not necessarily enhance the top-down control and stabilization. In the case where copepods are not omnivorous but only feed on microzooplankton, phytoplankton bloom occurs near the surface (see Fig.2), because the trophic control of primary producers becomes impeded. Moreover, for some ranges of $\epsilon_{P}$ and $\epsilon_{M}$ the species densities may exhibit oscillations (Fig.1). Our results show, however, that despite trophic pressure on the intermediate predator by the top predator-which is counterproductive from the point of view of efficient control of primary producers- stabilization of an eutrophic system is still possible. We also argue that the reported mechanism of top-down control should be quite general and could be applied to explain stability in other non-planktonic or even non-aquatic tri-trophic ecosystems. The necessary ingredients are the presence of pronounced environmental heterogeneity, fast movement of the top predator, and active switching of the top predator towards more abundant food sources. A phenomenon similar to self-shading of phytoplankton in the water column can also be seen in nonplanktonic systems. For instance, gradual depletion of a limiting nutrient by the primary producer may occur when there is a permanent flow of this nutrient through part of the environment occupied by this producer. As a result, the density of the nutrient in the direction of the flow will be given by integration of the consumption from the point of entrance to the given spacial location, i.e. similar to the integral term in the equation for $P$ in (4).

Among further interesting extension of the current research we can mention the following. Our conclusions about the coexistence of the three trophic levels were made based on particular parameterisations of 
the functional response of copepods: we used the Monod disk equation for a non-switching response and the kill-the-winner parametrisation for the response with an active switching. It is well known, however, that ecological models are very sensitive to the choice of the mathematical formulation of functional responses $[1,11,23]$ and the entire dynamics can change for a slightly different parametrization. Thus it would be interesting to verify whether or not the use of different parameterizations (see examples in [26]) of the same type of response would modify our results. It would be also important to explore the role of spatial heterogeneity of nutrients in the water column in the stability and coexistence of species. Even if the overall amount of nutrients is high and the ecosystem is eutrophic, depletion of nutrients in some parts of the euphotic zone, such as near the surface, and their accumulation in other parts (e.g. near the thermocline) can affect the dynamics $[17,18,47]$. This may require explicit modelling of the nutrient distribution in the water column. Finally, taking into account the impact of higher trophic levels (carnivorous zooplankton or piscivorous fish) on mesozooplankton may possibly ensure the coexistence of the three lower trophic levels, even for copepods with a non-switching functional response. Note, that the influence of higher levels can be modelled implicitly by introducing closure terms [19]. We are going to address the above issues in our future work.

\section{Acknowledgments}

We thank the Sergei Petrovskii (University of Leicester, UK) and Edward Codling (University of Essex, UK) for helpful comments and suggestions. 


\section{Appendix A}

In this section, we analyze the stationary states of the models in the well-mixing case. The model equations become

$$
\begin{aligned}
\frac{d \bar{P}(h, t)}{d t} & =r \bar{P}\left(1-\frac{\bar{P}}{K}\right)-m \bar{P}-f_{P_{1}}(\bar{P}) \bar{M}-f_{P_{2}}(\bar{P}, \bar{M}) \bar{Z} \\
\frac{d \bar{M}(h, t)}{d t} & =\gamma \bar{M} f_{P_{1}}(\bar{P})-\mu \bar{M}-f_{M}(\bar{P}, \bar{M}) \bar{Z} \\
\frac{d \bar{Z}(t)}{d t} & =\bar{Z}\left(\epsilon_{P} f_{P_{2}}(\bar{P}, \bar{M})+\epsilon_{M} f_{M}(\bar{P}, \bar{M})\right)-\delta \bar{Z}
\end{aligned}
$$

Note that to simplify analytical expressions we assume here that $\eta_{i}=1$.

The non-trivial stationary state $\left(\bar{P}^{*}>0, \bar{M}^{*}>0, \bar{Z}^{*}>0\right)$ is determined by the system

$$
\begin{aligned}
& 0=r \bar{P}^{*}\left(1-\frac{\bar{P}^{*}}{K}\right)-m \bar{P}^{*}-f_{P_{1}}\left(\bar{P}^{*}\right) \bar{M}-f_{P_{2}}\left(\bar{P}^{*}, \bar{M}^{*}\right) \bar{Z}^{*}, \\
& 0=\gamma \bar{M}^{*} f_{P_{1}}\left(\bar{P}^{*}\right)-\mu \bar{M}^{*}-f_{M}\left(\bar{P}^{*}, \bar{M}^{*}\right) \bar{Z}^{*}, \\
& 0=\left(\epsilon_{P} f_{P_{2}}\left(\bar{P}^{*}, \bar{M}^{*}\right)+\epsilon_{M} f_{M}\left(\bar{P}^{*}, \bar{M}^{*}\right)\right)-\delta,
\end{aligned}
$$

For Model 1, the stationary densities $\bar{P}^{*}, \bar{M}^{*}, \bar{Z}^{*}$ are given by (we omit the corresponding algebraic calculation):

$$
\bar{P}^{*}=\frac{-B \pm \sqrt{B^{2}-4 A C}}{2 A}, \quad \bar{M}^{*}=\frac{k_{2} \delta}{\epsilon_{M} \beta_{2}-\delta}, \bar{Z}^{*}=\left(\frac{\gamma g \bar{P}^{*}}{k_{1}+\bar{P}^{*}}-\mu\right) \frac{k_{2}+\bar{M} *}{\beta_{2}},
$$

where $A=r, B=k_{1} r-K r+m K, C=-k_{1} r K+m k_{1} K+g K \bar{M}^{*}$.

For Model 2, stationary densities $\bar{P}^{*}, \bar{M}^{*}, \bar{Z}^{*}$ are given by (we omit the corresponding algebraic calculation)

$$
\bar{P}^{*}=\frac{-B \pm \sqrt{B^{2}-4 A C}}{2 A}, \quad \bar{M}^{*}=\frac{k_{2} \delta}{\epsilon_{M} \beta_{2}-\delta}-\frac{\left(\epsilon_{M} \beta_{2}-\delta\right) P^{*}}{\epsilon_{P} \beta_{1}-\delta}, \bar{Z}^{*}=\left(\frac{\gamma g \bar{P}^{*}}{k_{1}+P^{*}}-\mu\right) \frac{k_{2}+\bar{P}^{*}+\bar{M}^{*}}{\beta_{2}}
$$

where $A=\left(\epsilon_{M} \beta_{2}-\delta\right) a, B=\epsilon_{P} \beta_{1}-\delta+\left(\epsilon_{M} \beta_{2}-\delta\right) b, C=\left(\epsilon_{M} \beta_{2}-\delta\right) c-\delta k_{2}$ with $a=-r /(g K)$, $b=1 /\left(\beta_{2} g K\right)\left(-\beta_{2} r k_{1}-\beta_{1} \gamma g K+\beta_{2} r K-\beta_{2} m K+\beta_{1} \mu K\right)$ and $c=1 /\left(\beta_{2} g K\right)\left(\beta_{2} r K k_{1}-\beta_{2} m K k_{1}+\beta_{1} \mu K k_{1}\right)$.

For Model 3, the equations for the stationary densities $\bar{P}^{*}, \bar{M}^{*}, \bar{Z}^{*}$ can not be found analytically and we numerically solved the corresponding system using Newton's method.

The stability analysis of the system was done by a standard linearization about $\left(\bar{P}^{*}, \bar{M}^{*}, \bar{Z}^{*}\right)$. We do not provide here the corresponding expressions for the elements of the Jacobian matrix for the sake of brevity.

We explore the feasibility of a non-trivial stationary state for each model and in the case the stationary state was positive, we checked its stability. For the considered parameter range we have not found any set of parameters where a non-trivial stationary state was stable.

\section{References}

[1] M.W. Adamson, A.Yu. Morozov. When can we trust our model predictions? Unearthing structural sensitivity in biological systems. Proc. R. Soc. A, 469 (2012), 20120500.

[2] P.A. Abrams, C.J. Walters. Invulnerable prey and the paradox of enrichment. Ecology, 77 (1996), $1125-1133$.

[3] A. Beckmann, I. Hense. Beneath the surface: characteristics of oceanic ecosystems under weak mixing conditions - a theoretical investigation. Prog. Oceanogr., 75 (2007), 771-796.

[4] M. N. Breckels, N. W. F. Bode, E. A. Codling, M. Steinke. The effect of grazing-mediated DMS production on the behaviour of the copepod Calanus helgolandicus. Mar. Drugs, 11 (2013), 2486-2500. 
[5] P.W. Boyd. Environmental factors controlling phytoplankton processes in the Southern Ocean. J. Phycol., 38 (2002), 844-861.

[6] P.W. Boyd, S.M. Smith, T. Cowles. Grazing patterns of copepods in the upwelling system off Peru. Limnol. Oceanogr., 25 (1980), 583-596.

[7] A. Calbet, F. Carlotti, R. Gaudy. The feeding ecology of the copepod Centropages typicus (Kroyer). Prog. Oceanogr., 72 (2007), 137-150

[8] F. Carlotti, and S. Nival Moulting and mortality rates of copepods related to age within stage: Experimental results. Mar. Ecol. Prog. Ser., 84 (1992), 235-243.

[9] F.P. Chavez, K.R. Buck, R.T. Barber. Phytoplankton taxa in relation to primary production in the equatorial Pacific. Deep Sea Res. 37 (1990), 1733-1752.

[10] P. Chow-Fraser, W.G. Sprules. Type-3 functional response in limnetic suspension-feeders, as demonstrated by in situ grazing rates. Hydrobiologia, 232 (1992), 175-191.

[11] F. Cordoleani, D. Nerini, M. Gauduchon, A. Morozov, J-C. Poggiale. Structural sensitivity of biological models revisited. J. Theor. Biol. 283 (2011), 82-91

[12] F. Courchamp, L. Berec, J. Gascoigne. Allee Effects in Ecology and Conservation. Oxford Uni. Press, Oxford, 2006.

[13] J.J. Cullen, M.R. Lewis, C.O. Davis, R.T. Barber. Photosynthetic characteristics and estimated growth rates indicate that grazing is the proximate control of primary production in the equatorial Pacific. J. Geophys. Res., 97(1992), 639-654

[14] B. Dennis. Allee effect: population growth, critical density, and chance of extinction. Nat. Resour. Model., 3 (1989), 481-538.

[15] E.G. Durbin, A.G. Durbin. Effects of temperature and food abundance on grazing and short-term weight change in the marine copepod Acartia hudsonica. Limnol. Oceanogr., 37 (1996), 361-378

[16] A. M. Edwards, J. Brindley. Zooplankton mortality and the dynamical behaviour of plankton population models. Bull. Math. Biol., 61 (1999), 303-339.

[17] C. A. Edwards, H. P. Batchelder, T. M. Powell. Modeling microzooplankton and macrozooplankton dynamics within a coastal upwelling system. J. Plankton Res., 22 (2000a), 1619-1648.

[18] C. A. Edwards, T. A. Powell, H. P. Batchelder. The stability of an NPZ model subject to realistic levels of vertical mixing. J. Mar. Res., 58 (2000b), 37-60.

[19] A.M. Edwards, A Yool. The role of higher predation in plankton population models. J. Plankton Res., 22 (2000), $1085-1112$

[20] K. F. Edwards, M. K. Thomas, C. A. Klausmeier, E. Litchman. Light and growth in marine phytoplankton: Allometric, taxonomic, and environmental variation. Limnol. Oceanogr., 60 (2015), 540-552

[21] J.Z. Farkas, A. Yu. Morozov, E.G. Arashkevich, A. Nikishina. Revisiting the stability of spatially heterogeneous predatorprey systems under eutrophication. Bull. Math. Biol., 77 (2015), 1886-1908.

[22] B.W. Frost. Grazing control of phytoplankton stock in the open subarctic Pacific Ocean: a model assessing the role of mesozooplankton, particularly the large calanoid copepods Neocalanus spp. Mar. Ecol. Prog. Ser., 39 (1987), 49-68.

[23] G.F. Fussmann, B. Blasius. Community response to enrichment is highly sensitive to model structure. Biol. Lett., $1(2005), 9-12$

[24] A. Gabric, N. Murray, L. Stone, M. Kohl. Modelling the production of dimethylsulfide during a phytoplankton bloom. J. Geophys. Res., 98 (1993), 22805-22816.

[25] M. Genkai-Kato, N. Yamamura. Unpalatable prey resolves the paradox of enrichment. P. Roy. Soc. Lond. B. Bio., 266, (1999), 1215-1219

[26] W. Gentleman, A. Leising, B. Frost, S. Storm, J. Murray. Functional responses for zooplankton feeding on multiple resources: a review of assumptions and biological dynamics. Deep-sea Res. Pt. II, 50 (2003), 2847-2875.

[27] I. Gismervik, I. Top-down impact by copepods on ciliate numbers and persistence depends on copepod and ciliate species composition. J. Plankton Res., 28 (2006), 499-507.

[28] I. Gismervik, T. Andersen. Prey switching by Acartia clausi: experimental evidence and implications of intraguild predation assessed by a model. Mar. Ecol. Prog. Ser., 157(1997), 247-259

[29] P. J. Hansen, P.K. Bjornsen, B.W Hansen. Zooplankton grazing and growth: Scaling within the 2-2000 $\mu$ m body size range. Limnol. Oceanogr., 42 (1997), 687-704.

[30] F. C. Hansen, M. Reckermann, W. C. M. Klein Breteler. Phaeocystis blooming enhanced by copepod predation on protozoa: evidence from incubation experiments. Mar. Ecol. Prog. Ser., 102 (1993), 51-57.

[31] C.X.J. Jensen, L.R. Ginzburg. Paradoxes or theoretical failures? The jury is still out. Ecol. Model., 188 (2005), 3-14.

[32] A. Kharab, R. B. Guenther. An Introduction to Numerical Methods: A MATLAB Approach. Third edition. CRC Press, Boca Raton, 2012.

[33] T. Kierboe, Saiz, M. Viitasalo. Prey switching behaviour in the planktonic copepod Acartia tonsa. Mar. Ecol. Prog. Ser., 143(1996), 65-75.

[34] W. Lampert. Vertical distribution of zooplankton: density dependence and evidence for an ideal free distribution with costs. BMC Biol., 3 (2005), 10.

[35] N. Lewis, A. Morozov, M. Breckels, M. Steinke, and E. Codling. Multitrophic interactions in the sea: assessing the effect of infochemical-mediated foraging in a 1-d spatial model. MMNP, 8(2013), 25-44.

[36] K.M. Meyer, M. Vos, W. M. Mooij, W. H. G. Hol, A. J. Termorshuizen, W. H. van der Putten. Testing the paradox of enrichment along a land use gradient in a multitrophic aboveground and belowground community. PLoS ONE 7 (2012): e49034. 
[37] A. Yu. Morozov, M. Sen, M. Banerje. Top-down control in a patchy environment: Revisiting the stabilizing role of food-dependent predator dispersal. Theor. Popul. Biol., 81 (2012), 9-19.

[38] A. Yu. Morozov. Incorporating complex foraging of zooplankton in models: role of micro and mesoscale processes in macroscale patterns. In Dispersal, individual movement and spatial ecology: a mathematical perspective (eds M Lewis, P Maini \& S Petrovskii), pp. 1-10. New York, NY: Springer, 2011.

[39] A. Yu. Morozov, E.G. Arashkevich, A. Nikishina, K Solovyev. Nutrient-rich plankton communities stabilized via predator-prey interactions: revisiting the role of vertical heterogeneity. Math. Med. Biol., 28 (2011), 185-215

[40] A. Yu. Morozov, A. F. Pasternak, E. G. Arashkevich. Revisiting the role of individual variability in population persistence and stability. PLoS ONE, (8) (2013), e70576.

[41] A. Yu. Morozov, S.V. Petrovskii. Feeding on multiple sources: towards a universal parameterization of the functional response of a generalist predator allowing for switching. PLoS ONE, (8) (2013), e74586. doi: 10.1371/journal.pone.0074586.

[42] A. Mougi, K. Nishimura. A resolution of the paradox of enrichment. J. Theor. Biol., 248, (2007), 194-201

[43] W.W. Murdoch. The functional response of predators. J. Appl. Ecol., 10 (1973), 335-342

[44] S. Petrovskii S, B. Li, H. Malchow. Transition to spatiotemporal chaos can resolve the paradox of enrichment. Ecol. Complex, 1 (2004), 37-47.

[45] G.A. Polis, R.D. Holt. 1992. Intraguild predation: the dynamics of complex trophic interactions. Trends. Ecol. Evol., 7 (1992), 151-155

[46] J.E.G. Raymont. Plankton and Productivity in the Oceans. Phytoplankton, vol. 1Pergamon. Oxford (1980)

[47] A. B. Ryabov, L. Rudolf, B. Blasius. Vertical distribution and composition of phytoplankton under the influence of an upper mixed layer. J. Theor. Biol., 263 (2010), 120-133.

[48] A. B. Ryabov, A. Morozov, B. Blasius Imperfect prey selectivity of predators promotes biodiversity and irregularity in food webs. Ecol. Letts. , 18.11 (2015), 1262-1269.

[49] M. L. Rosenzweig. Paradox of enrichment: destabilization of exploitation ecosystems in ecological time. Science, 171 (1971), 385-387.

[50] M. L. Rosenzweig, R. H. MacArthur. Graphical representation and stability conditions of predator-prey interactions. Am. Nat., 97 (1963), 209-223.

[51] S. Roy, J. Chattopadhyay. The stability of ecosystems: a brief overview of the paradox of enrichment. J. Bioscience, 32(2007), 421-428.

[52] P.A. Stephens, W.J. Sutherland. Consequences of the Allee effect for behaviour, ecology and conservation. Trends. Ecol. Evol., 14 (1999), 401-405.

[53] D.K. Stoecker, J.M. Capuzzo. Predation on Protozoa: its importance to zooplankton. J. Plankton Res., 12 (1990), 891-908

[54] W. K. Tang, and M. Taal Trophic modification of food quality by heterotrophic protists: species-specific effects on copepod egg production and egg hatching. J. Exp. Mar. Biol. Ecol., 318.1 (2005), 85-98.

[55] P. Tiselius, P.R. Jonsson. Foraging behaviour of six calanoid copepods: observations and hydrodynamic analysis. Mar. Ecol. Prog. Ser., 66(1990), 23-33

[56] S. M. Vallina, B. Ward, S. Dutkiewicz, and M. Follows. Maximal feeding with active preyswitching: A kill-the-winner functional response and its effect on global diversity and biogeography, Prog. Oceanogr., 120 (2014), 93-109.

[57] L. van Duren, J. Videler, J. Swimming behaviour of developmental stages of the calanoid copepod Temora longicornis at different food concentrations. Mar. Ecol. Prog. Ser., 126 (1995), 153-161. 